\title{
Aumento das internações por uso de drogas de abuso: destaque para mulheres e idosos
}

\author{
Increase in hospital admissions for drug use in \\ Brazil particularly in women and elderly \\ Thamires Fernandes Cardoso da Silva Rodrigues' \\ https://orcid.org/0000-0001-7942-4989 \\ Rosana Rosseto de Oliveira \\ https://orcid.org/0000-0003-3373-1654 \\ Maria das Neves Decesaro 1 \\ https://orcid.org/0000-0003-2275-5415 \\ Thais Aidar de Freitas Mathias \\ https://orcid.org/0000-0002-2853-1634
}

\section{Palavras-chave \\ Abuso de drogas, \\ hospitalização, usuários de drogas, psiquiatria.}

\section{RESUMO}

Objetivo: Analisar a tendência das taxas de internação por transtornos mentais e comportamentais devido ao uso de drogas no Brasil. Métodos: Estudo ecológico de séries temporais, das internações de residentes no Brasil, no período de 2005 a 2015, registradas no Sistema de Informações Hospitalares do Sistema Único de Saúde. Realizou-se análise de tendência e diferença relativa das taxas, por triênios extremos e do modelo de regressão polinomial para todo o período. Resultados: As taxas de internação por uso de drogas aumentaram em média 0,96 ao ano, com declínio após o ano de 2012. A região Norte exibiu tendência crescente para ambos os sexos (0,56 ao ano) e na região Nordeste, o sexo masculino apresentou tendência decrescente (-0,33 ao ano). Em relação às mulheres, houve aumento das taxas em todas as faixas etárias. Detectou-se aumento nas taxas de internação entre idosos, e a região Norte apresentou diferença relativa mais expressiva (4,7 para homens e 2,7 para mulheres). Conclusão: A tendência das taxas de internação devidas ao uso de drogas mostrou-se crescente, com queda da curva de tendência apenas ao final do período. As mulheres e os idosos tiveram aumento das taxas de internação em relação aos períodos iniciais e finais da amostra.

\section{ABSTRACT}

Objective: To analyze the trend of hospitalization rates for mental and behavioral disorders due to drug use in Brazil. Methods: Ecological study of time series of hospitalizations of Brazilian residents, from 2005 to 2015, recorded in the Hospital Information System of the Unified Health System. A trend analysis and relative difference in rates were performed for extreme triennial and of the polynomial regression model for the whole period. Results: Rates of hospitalization for drug use increased by an average of 0.96 per year, with a decline after 2012. The North region showed a growing tendency for both sexes ( 0.56 per year) and in the Northeast region, the males showed a decreasing trend ( -0.33 per year). In relation to women,

1 Universidade Estadual de Maringá, Programa de Pós-Graduação, Maringá, PR, Brasil.

Recebido em

9/Mar/2019

Aprovado em 


\section{Keywords}

Drug abuse, hospitalization, drug users, psychiatry. rates increased in all age groups. There was an increase in hospitalization rates among the elderly, the North region presented a more significant relative difference (4.7 for men and 2.7 for women). Conclusion: The trend of hospitalization rates due to drug use showed to be increasing with trend curve only at the end of the period. Women and the elderly had an increase in hospitalization rates in relation to the initial and final periods of the sample.

\section{INTRODUÇÃO}

O consumo de drogas de abuso revela-se um grande desafio para a saúde pública mundial. Estima-se que 1 bilhão de pessoas no mundo, com idade entre 15 e 64 anos, utilizaram drogas pelo menos uma vez em 2015. Dessas, 29,5 milhões desenvolveram transtornos mentais que resultaram na perda de 28 milhões de anos de vida saudáveis, devido às incapacidades e mortes prematuras'.

A literatura destaca que $5 \%$ das internações que ocorrem em países desenvolvidos estão relacionadas ao consumo de drogas². Na Coreia do Sul, os transtornos mentais ocasionados pela dependência de drogas consistem na sétima causa de doenças não fatais que comprometem a qualidade de vida das pessoas. Esse agravo incide principalmente nas crianças, adolescentes e mulheres jovens, com idade entre 20 e 29 anos $^{3}$. Nos Estados Unidos, a dependência de opiáceos corresponde ao segundo transtorno mental com maior mortalidade 4 . O Brasil não se difere desse cenário, visto que, no ano de 2013, em torno de 47\% da população acima de 16 anos manteve contato com as drogas ilícitas, duas a cada dez tiveram acesso à cocaína e, dessas, 5\% a utilizaram 5 .

Historicamente, a atenção aos transtornos mentais e à dependência de drogas no país possuía apenas os hospitais psiquiátricos como modelo de tratamento, cujas características eram asilares e higienistas, desconsiderando o sujeito enquanto pessoa ${ }^{6}$. $O$ atendimento passou por modificações, principalmente a partir da Lei no 10.216, de 6 de abril de 2001, conhecida como Lei da Reforma Psiquiátrica. Essa lei determinou que a assistência à saúde mental se estruturasse de forma integral e humanizada, livre de qualquer discriminação, buscando minimizar a necessidade de internações ${ }^{7}$. A partir dela, outros avanços ocorreram, instituindo a política de "desinstitucionalização" e fomentando a redução de leitos em hospitais psiquiátricos e a concomitante criação de serviços substitutivos, tais como os Centros de Atenção Psicossocial (CAPS) e leitos psiquiátricos em hospitais gerais?.

Dessa forma, como alternativa à internação psiquiátri$\mathrm{ca}$, o atendimento às situações de urgência, emergência e crise deve ocorrer nos Centros de Atenção Psicossocial Álcool e Drogas III (CAPS-ad III) e nos hospitais gerais, os quais consideram as reais demandas dos sujeitos e suas famílias, possibilitando a integração social e a continuidade do cuidado9. Entretanto, nota-se que há desigualdade na expansão desses serviços entre os estados brasileiros, devido às fortes políticas proibicionistas quanto ao uso de drogas, à força do modelo manicomial e higienista presente em algumas localidades $^{8,9}$. Assim, apesar dos esforços em substituir o modelo hospitalocêntrico para o comunitário, este ainda continua em processo de efetivação no Brasil|10,11.

Ressalta-se a importância de conhecer o perfil epidemiológico e a tendência das internações decorrentes de drogas de abuso, a fim de promover a melhoria da assistência, direcionar adequadamente os recursos financeiros e avaliar o impacto das políticas públicas. Elevadas taxas de hospitalização podem indicar, a princípio, o aumento da prevalência do abuso de drogas na população, também a sua cronicidade, que eleva a demanda e sucessivos atendimentos tanto por serviços ambulatoriais quanto por leitos hospitalares. Assim, analisar a demanda por internações pode ser uma forma de

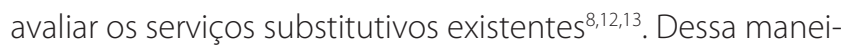
ra, o objetivo deste estudo foi analisar a tendência das taxas de internação por transtornos mentais e comportamentais devidos ao uso de drogas no Brasil.

\section{MÉTODOS}

Trata-se de um estudo ecológico, de séries temporais, das internações por transtornos mentais devidos ao uso de drogas no Brasil, no período de 2005 a 2015. Para a escolha do período de estudo, considerou-se que até 2005 o consumo de drogas de abuso manteve-se discreto e estável no Brasil, com crescimento posterior, associando-se a diversos agravos de saúde, a criminalidade e a violência ${ }^{14}$.

O Brasil possui 204 milhões de habitantes, com 101 miIhões de homens e 103 milhões de mulheres. O país compõe-se por 27 unidades federativas, agrupadas em cinco grandes regiões: Norte, Nordeste, Sudeste, Sul e Centro-Oeste $^{14}$. Para este estudo, o Distrito Federal foi analisado em conjunto com a região Centro-Oeste.

Analisaram-se os registros de internações por uso de drogas, disponíveis no Sistema de Informações Hospitalares do Sistema Único de Saúde (SIH/SUS), de residentes com idade entre 1 ou mais anos de idade. Filtraram-se os dados por meio da lista de morbidade da Classificação Internacional de Doenças, em sua 10a Revisão (CID-10), selecionando-se somente hospitalizações com diagnóstico principal no Capítulo V (Transtornos Mentais e Comportamentais - Códigos de F00 a F99). Após, escolheram-se apenas as internações com 
diagnóstico principal no agrupamento de transtornos mentais e comportamentais devidos ao uso de drogas, codificados entre F10 e F19, excluindo-se as internações resultantes do consumo estrito de álcool (F10) e aquelas cujo sexo foi ignorado, conforme consta na figura 1.

As taxas de internação foram calculadas por meio da razão entre o número de hospitalizações por uso de drogas e a população correspondente, segundo sexo, idade, ano de internação e região de residência (multiplicados por 100 mil habitantes). A população residente de cada local, faixa etária e ano foi obtida por meio dos registros do Instituto Brasileiro de Geografia e Estatística ${ }^{15}$.
A análise de tendência das taxas de internação foi realizada pelo modelo de regressão linear polinomial. Consideraram-se as taxas de internações por uso de drogas como variável dependente $(Y)$ e os anos de estudo como variável independente $(X)$. Primeiramente, realizaram-se diagramas de dispersão, com posterior suavização da série por meio do cálculo da média móvel. Testaram-se os modelos de regressão linear simples $(y=\beta 0+\beta 1 X)$ e, quando necessário, modelos de segunda ordem $\left(y=\beta 0+\beta 1 X+\beta 2 X^{2}\right)$. Considerou-se como melhor modelo polinomial aquele que apresentou significância estatística, com melhor coeficiente de determinação e resíduo sem vício.

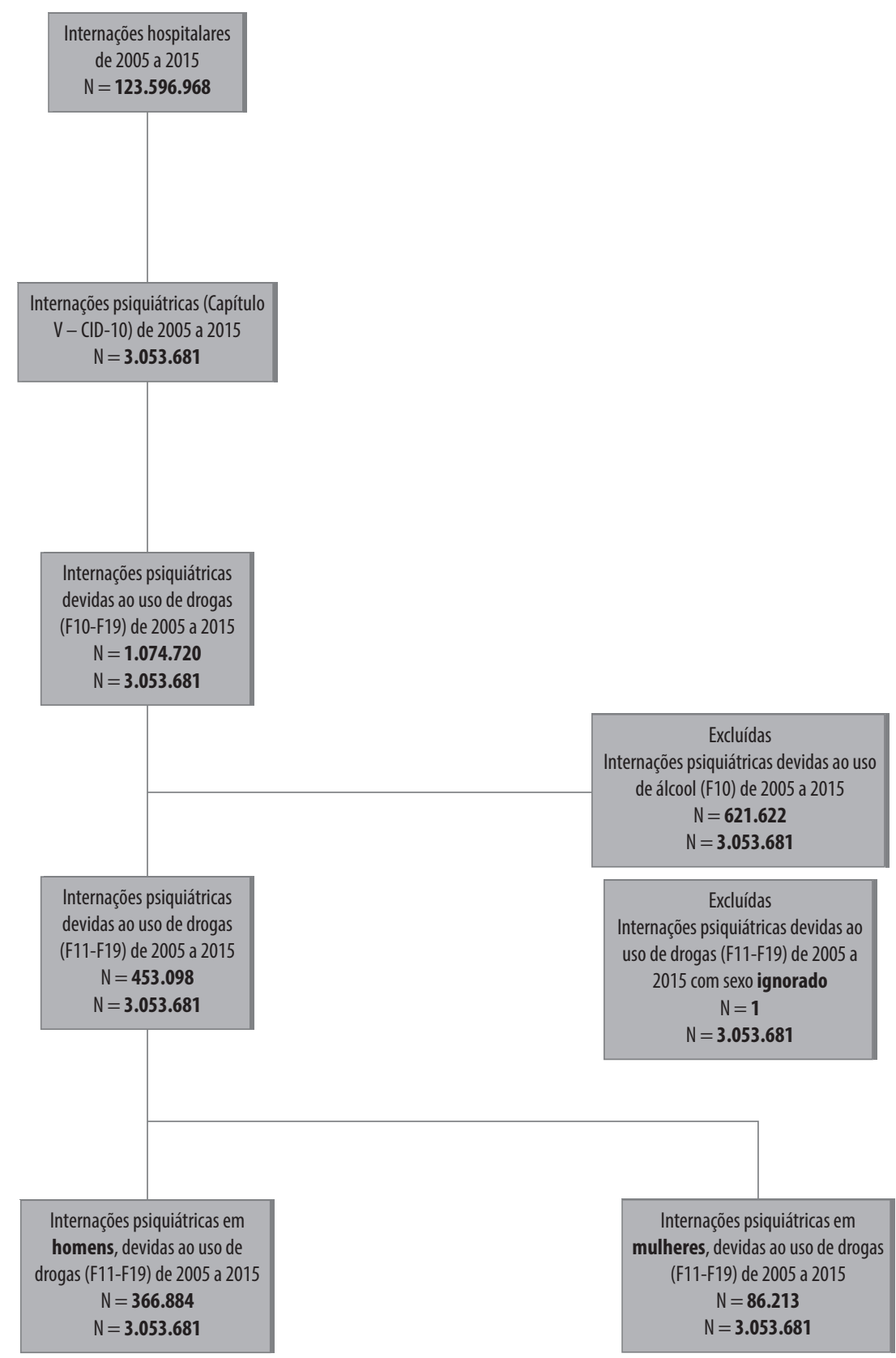

Figura 1. Processo de identificação e seleção das internações hospitalares do Sistema de Informações Hospitalares do Sistema único de Saúde (SIH/SUS) por uso de drogas - Brasil, 2005 a 2015. 
Quando dois modelos se apresentaram semelhantes, optou-se pelo modelo mais simples, ou seja, de menor ordem. As análises foram realizadas por meio do software SPSS, versão 20.1.

Utilizou-se ainda o cálculo de diferença relativa [(V2 - V1)/ V1] entre as taxas de internações ocorridas nos triênios iniciais (2005 a 2007) e finais (2013 a 2015). Para a apresentação gráfica das hospitalizações por meio de mapas temáticos, as taxas foram apresentadas em quintis para os dois triênios, de acordo com o número máximo e mínimo, utilizando-se escalas de cor cinza, com a cor branca para as taxas mais baixas e a cor preta para as mais elevadas.

O estudo seguiu em consonância com as diretrizes disciplinadas pelo Conselho Nacional de Saúde do Ministério da Saúde (Resolução no 466/2012) ${ }^{16}$, recebendo dispensa da avaliação do Comitê Permanente de Ética em Pesquisa com Seres Humanos da Instituição Signatária, sob ofício de no 17/2016.

\section{RESULTADOS}

No Brasil, de 2005 a 2015, ocorreram 123.596.968 internações, das quais $0,37 \%$ foi devido ao uso de drogas. A análise de tendência revelou que as taxas de internações se mantiveram crescentes até o ano de 2012 (aumento médio de 0,96 ao ano; $\left.r^{2}=0,98\right)$, com posterior declínio. A região Norte exibiu tendência crescente para ambos os sexos e em todo o período, com aumento médio na taxa de internação de 0,56 ao ano $(p=0,001)$. Já na região Nordeste, o sexo masculino apresentou tendência decrescente, com redução média de 0,33 ao ano $\left(r^{2}=0,69\right)$ (Tabela 1$)$.

Observou-se que as internações por uso de drogas ocorreram majoritariamente na população com idade entre 20 e 39 anos. Entretanto, detectou-se aumento nas taxas de internação entre os idosos, com diferença relativa geral, entre os triênios estudados, de 0,4 para as mulheres e 0,2 para os homens. A região Norte apresentou a taxa de internação mais expressiva de indivíduos com 60 anos ou mais (diferença relativa de 4,7 para homens e 2,7 para mulheres), e a região Centro-Oeste exibiu diminuição das taxas entre os adolescentes (-0,3 e -0,2 nas mulheres e homens, respectivamente) (Tabela 2).

Verificou-se ainda que, comparado aos homens, o crescimento das taxas de internação entre o primeiro e o segundo triênio, em todas as faixas etárias, foi superior para as mulheres (Tabela 2). Quanto à distribuição das taxas de internações devidas ao uso de drogas no sexo feminino, notou-se que no primeiro triênio as regiões Sudeste, Sul e Centro-Oeste concentraram as maiores taxas. Já no último triênio, observou-se aumento em alguns estados do Norte, como Acre e Tocantins, os quais inicialmente exibiam taxas de internação inferiores a 3 por 100 mil habitantes (2,5 para o Acre e 2,88 para o Tocantins), saltando para 20,7 e 17,11 por 100 mil habitantes, respectivamente (Figura 2).

Tabela 1. Tendência das taxas de internação (por 100.000 habitantes) por transtornos mentais e comportamentais devidos ao uso de drogas, segundo sexo - Brasil e grandes regiões, 2005 a 2015

\begin{tabular}{|c|c|c|c|c|}
\hline & Modelo & $R^{2}$ & $p$ & Tendência* \\
\hline Brasil & $y=24,68+0,96 x-0,40 x^{2}$ & 0,98 & $<0,001$ & $\uparrow$ \\
\hline Feminino & $y=9,8+0,61 x-0,17 x^{2}$ & 0,95 & $<0,001$ & $\uparrow$ \\
\hline Masculino & $y=40,34+1,32 x-0,64 x^{2}$ & 0,98 & $<0,001$ & $\uparrow$ \\
\hline Norte & $y=3,31+0,56 x$ & 0,85 & $<0,001$ & $\uparrow$ \\
\hline Feminino & $y=1,52+0,22 x$ & 0,87 & $<0,001$ & $\uparrow$ \\
\hline Masculino & $y=4,81+0,78 x$ & 0,80 & 0,001 & $\uparrow$ \\
\hline Nordeste & $y=12,55-0,14 x-0,17 x^{2}$ & 0,76 & 0,006 & $\uparrow$ \\
\hline Feminino & $y=4,27+0,05 x-0,09 x^{2}$ & 0,91 & $<0,001$ & $\uparrow$ \\
\hline Masculino & $y=21,05-0,33 x-0,26 x^{2}$ & 0,69 & 0,012 & $\downarrow$ \\
\hline Sudeste & $y=22,70+0,95 x-0,37 x^{2}$ & 0,92 & $<0,001$ & $\uparrow$ \\
\hline Feminino & $y=9,42+0,77 x-0,13 x^{2}$ & 0,92 & $<0,001$ & $\uparrow$ \\
\hline Masculino & $y=36,33+1,16 x-0,60 x^{2}$ & 0,93 & $<0,001$ & $\uparrow$ \\
\hline Sul & $y=64,68+3,53 x-1,14 x^{2}$ & 0,99 & $<0,001$ & $\uparrow$ \\
\hline Feminino & $y=23,12+1,55 x-0,52 x^{2}$ & 0,94 & $<0,001$ & $\uparrow$ \\
\hline Masculino & $y=107,11+5,56 x-1,77 x^{2}$ & 0,99 & $<0,001$ & $\uparrow$ \\
\hline Centro-0este & $28,89+0,64 x-0,59 x^{2}$ & 0,93 & $<0,001$ & $\uparrow$ \\
\hline Feminino & $10,39+0,30 x-0,21 x^{2}$ & 0,95 & $<0,001$ & $\uparrow$ \\
\hline Masculino & $47,46+0,95 x-0,98 x^{2}$ & 0,93 & $<0,001$ & $\uparrow$ \\
\hline
\end{tabular}

* $\uparrow$ Crescente; $\downarrow$ Decrescente. 
Tabela 2. Internações por transtornos mentais e comportamentais devidos ao uso de drogas segundo sexo e idade (no, \% e taxa), por triênios - Brasil e grandes regiões, 2005 a 2015

\begin{tabular}{|c|c|c|c|c|c|c|c|c|c|c|}
\hline & \multicolumn{4}{|c|}{ Feminino } & \multirow{3}{*}{$\begin{array}{l}\text { Dif. relativa das } \\
\text { taxas }\end{array}$} & \multicolumn{4}{|c|}{ Masculino } & \multirow{3}{*}{$\begin{array}{l}\text { Dif. relativa das } \\
\text { taxas }\end{array}$} \\
\hline & \multicolumn{2}{|c|}{ 2005-2007 } & \multicolumn{2}{|c|}{ 2013-2015 } & & \multicolumn{2}{|c|}{ 2005-2007 } & \multicolumn{2}{|c|}{ 2013-2015 } & \\
\hline & $\mathrm{N}$ & Taxa & $\mathrm{N}$ & Taxa & & $\mathrm{N}$ & Taxa & $\mathrm{N}$ & Taxa & \\
\hline \multicolumn{11}{|l|}{ Brasil } \\
\hline$<19$ anos & 2.964 & 2,9 & 4.157 & 4,3 & 0,5 & 10.002 & 9,6 & 12.511 & 12,6 & 0,3 \\
\hline 20-39 anos & 7.920 & 8,5 & 17.698 & 17,6 & 1,1 & 50.038 & 53,3 & 74.352 & 73,4 & 0,4 \\
\hline 40-59 anos & 2.001 & 3,3 & 5.304 & 7,3 & 1,2 & 8.983 & 15,7 & 19.406 & 28,1 & 0,8 \\
\hline 60 ou mais & 290 & 1,0 & 528 & 1,4 & 0,4 & 700 & 3,1 & 1.091 & 3,6 & 0,2 \\
\hline \multicolumn{11}{|l|}{ Norte } \\
\hline$<19$ anos & 45 & 0,4 & 106 & 1,0 & 1,5 & 90 & 0,9 & 302 & 2,8 & 2,1 \\
\hline 20-39 anos & 105 & 1,4 & 486 & 5,6 & 3,0 & 371 & 4,9 & 1.696 & 18,9 & 2,8 \\
\hline 40-59 anos & 27 & 0,8 & 240 & 5,1 & 5,4 & 70 & 1,9 & 528 & 10,8 & 4,7 \\
\hline 60 ou mais & 8 & 0,6 & 39 & 2,2 & 2,7 & 8 & 0,6 & 59 & 3,4 & 4,7 \\
\hline \multicolumn{11}{|l|}{ Nordeste } \\
\hline$<19$ anos & 536 & 1,7 & 495 & 1,7 & - & 1.869 & 5,8 & 1.601 & 5,3 & $-0,1$ \\
\hline 20-39 anos & 1.170 & 4,5 & 1.747 & 6,1 & 0,3 & 9.728 & 37,4 & 9.716 & 34,4 & $-0,1$ \\
\hline 40-59 anos & 270 & 1,8 & 494 & 2,7 & 0,5 & 1.359 & 10,0 & 2.297 & 13,7 & 0,4 \\
\hline 60 ou mais & 44 & 0,6 & 60 & 0,6 & - & 80 & 1,4 & 176 & 2,4 & 0,7 \\
\hline \multicolumn{11}{|l|}{ Sudeste } \\
\hline$<19$ anos & 1.252 & 3,2 & 1.580 & 4,3 & 0,3 & 3.343 & 8,3 & 3.877 & 10,2 & 0,2 \\
\hline 20-39 anos & 3.602 & 9,0 & 8.382 & 20,2 & 1,2 & 19.648 & 49,2 & 26.635 & 63,5 & 0,3 \\
\hline $40-59$ anos & 793 & 2,8 & 2.600 & 7,8 & 1,8 & 3.707 & 13,9 & 7.728 & 24,7 & 0,8 \\
\hline 60 ou mais & 88 & 0,7 & 246 & 1,3 & 0,8 & 226 & 2,2 & 490 & 3,4 & 0,5 \\
\hline \multicolumn{11}{|l|}{ Sul } \\
\hline$<19$ anos & 848 & 6,3 & 1.774 & 14,3 & 1,3 & 3.771 & 26,9 & 5.966 & 46,0 & 0,7 \\
\hline $20-39$ anos & 2.351 & 17,9 & 5.764 & 41,7 & 1,7 & 16.165 & 122,1 & 30.354 & 215,2 & 0,8 \\
\hline 40-59 anos & 778 & 7,9 & 1.603 & 14,1 & 0,8 & 3.358 & 35,8 & 7.665 & 70,7 & 1,0 \\
\hline 60 ou mais & 120 & 2,6 & 154 & 2,4 & $-0,1$ & 333 & 9,1 & 298 & 5,8 & $-0,4$ \\
\hline \multicolumn{11}{|l|}{ Centro-0este } \\
\hline$<19$ anos & 283 & 3,9 & 202 & 2,8 & $-0,3$ & 929 & 12,3 & 765 & 10,2 & $-0,2$ \\
\hline $20-39$ anos & 692 & 9,8 & 1.319 & 16,6 & 0,7 & 4.126 & 58,4 & 5.951 & 74,5 & 0,3 \\
\hline 40-59 anos & 133 & 3,2 & 367 & 6,8 & 1,1 & 489 & 12,2 & 1.188 & 22,9 & 0,9 \\
\hline 60 ou mais & 30 & 2,0 & 29 & 1,3 & $-0,3$ & 53 & 3,8 & 68 & 3,4 & $-0,1$ \\
\hline
\end{tabular}

Referente ao sexo masculino, a região Sul concentrou grande parte das internações, com taxa média de 56,3 por 100 mil habitantes no primeiro triênio e de 94,2 internações por 100 mil habitantes no último. Destaca-se ainda o Rio Grande do Sul, com taxa de 51,2 entre 2005 e 2007 e de 157,9 de 2013 a 2015. Observou-se também acréscimo importante das taxas de internações no estado do Acre, passando de 9,5 internações/100 mil habitantes no primeiro triênio para 105,4 internações/100 mil habitantes no último triênio de estudo (Figura 2).

\section{DISCUSSÃO}

O estudo possibilitou conhecer a tendência e a distribuição das internações por transtornos mentais e comportamentais devidas ao uso de drogas nas cinco regiões brasileiras. Os resultados evidenciam a tendência de aumento das internações psiquiátricas por uso de drogas e de declínio apenas ao final do período analisado para grande parte do país, com exceção da região Norte, que se manteve crescente. Constatou-se ainda aumento das taxas de internações por drogas 


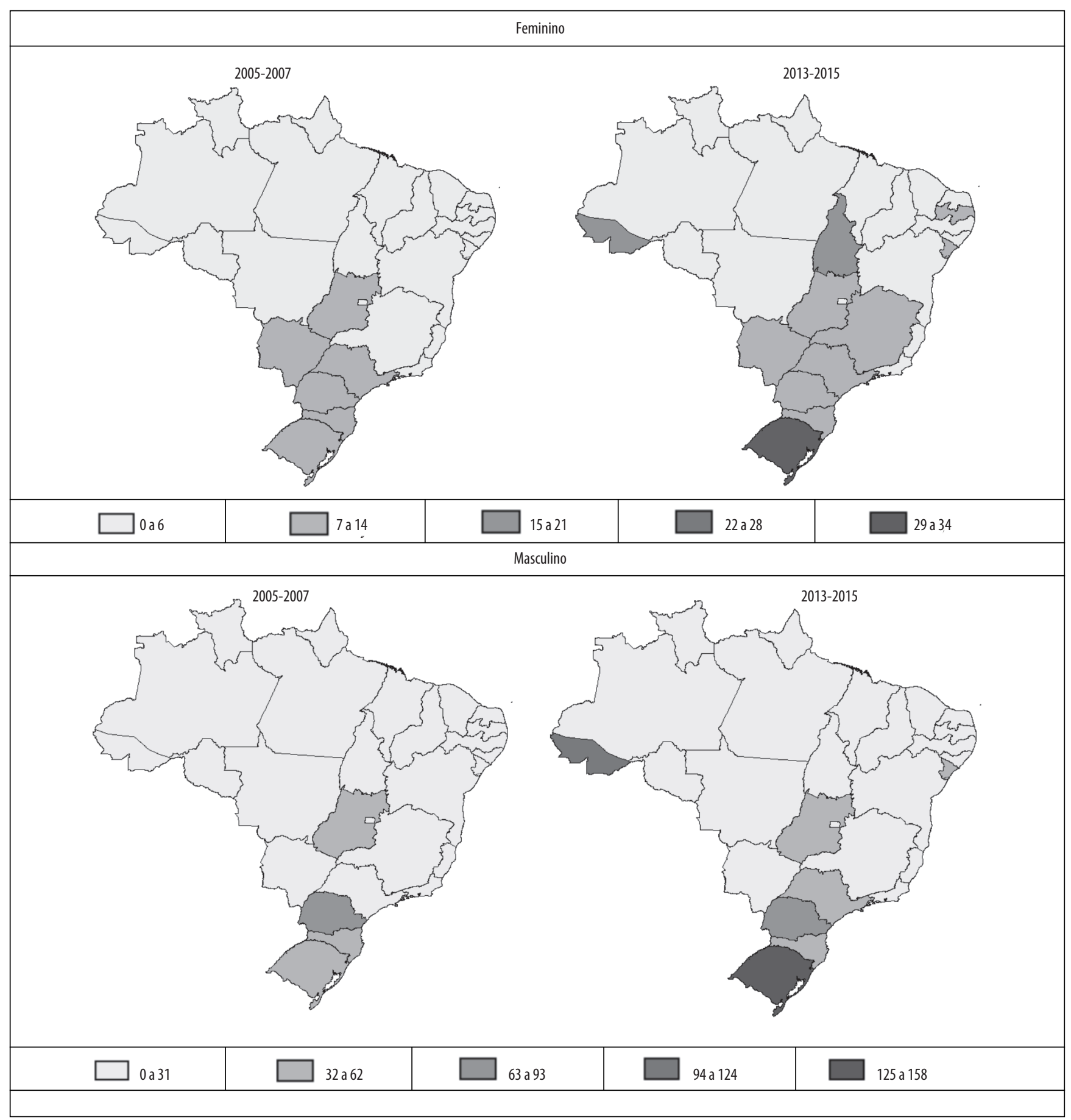

Figura 2. Distribuição das taxas de internação (por 100.000 habitantes) por transtornos mentais e comportamentais devidos ao uso de drogas, segundo sexo e unidades federativas - Brasil, 2005 a 2015.

de abuso entre idosos e mulheres, com discreta diferença entre as regiões do país.

O aumento das taxas de internação pode estar relacionado ao crescimento do consumo de drogas pelas pessoas em geral, independentemente da classe econômica e da faixa etária ${ }^{11,17}$. O aumento do consumo de drogas e a utilização de serviços de saúde vêm sendo relatados em diversos países¹. O Relatório Mundial de Drogas emitido em 2017 apon- tou para o aumento no consumo de cannabis na América do Norte e na Europa Central, e de cocaína na América do Sul'.

Uma pesquisa desenvolvida no Canadá identificou que os usuários de drogas utilizam com maior frequência os serviços de emergência hospitalar, aproximadamente de sete a oito vezes mais que o restante da população ${ }^{18}$. Um estudo de coorte conduzido na França detectou que 4\% das hospitalizações por uso de drogas resultaram em morte ou em ad- 
missão em unidades de terapia intensiva, e elas foram principalmente associadas ao consumo de opiáceos, cocaína e narcóticos ${ }^{19}$. Vale considerar ainda que usuários de drogas têm maior probabilidade de ser hospitalizados e passar por reinternações, devido a exposição à violência, acidentes de trânsito, overdose, desnutrição e ao risco de contrair infecções, tais como pneumonia, tuberculose e HIV2,18.

Em contrapartida, no que se refere à diminuição das taxas de internações por uso de drogas, detectadas apenas nos últimos anos do presente estudo, tal redução pode ser resultado dos esforços do Brasil e do mundo em promover a melhoria da assistência dispensada às pessoas com transtornos mentais. Para tanto, o Ministério da Saúde brasileiro fortaleceu as políticas públicas, a fim de redirecionar o modelo assistencial, substituindo progressivamente os leitos hospitalares psiquiátricos por uma rede de Atenção Psicossocial ${ }^{20}$. Assim, em 2005, o país detinha 0,23 leito psiquiátricos públicos para cada mil habitantes, reduzindo para 0,17 leito por mil habitantes em hospitais psiquiátricos em $2013^{15}$.

Apesar dessa redução, tal índice revela-se alarmante, pois se trata da média nacional e há alguns municípios que detêm grande número de hospitais psiquiátricos ou que mantêm as hospitalizações e a abstinência forçada como único recurso para o tratamento da dependência ${ }^{21}$. Vale ressaltar que o processo de redução de leitos em hospitais psiquiátricos e a desospitalização dos sujeitos com transtornos mentais prosseguiram de maneira distinta nas regiões brasileiras, variando conforme a adesão à Reforma Psiquiátrica, as transformações culturais e as pactuações governamentais locais²1. A diminuição dos leitos deve ser acompanhada pelo fortalecimento dos serviços de base comunitária, como os CAPS, com terapêuticas mais resolutivas que aliam o suporte extra ao intra-hospitalar, ambos respeitando as especificidades de cada sujeito e de suas famílias ${ }^{12}$.

No tocante à disparidade entre as regiões, sabe-se que o país possui dimensões continentais, desse modo há grande diversidade cultural e socioeconômica, inclusive quanto à disponibilidade de serviços de saúde que atendam às necessidades da população ${ }^{22}$. As regiões Sul e Sudeste possuem mais recursos econômicos e acesso aos serviços de saúde, contrariamente ao Norte e ao Nordeste, os quais demonstram maior vulnerabilidade quantos aos indicadores socioeconômicos e de morbimortalidade ${ }^{23}$. Pesquisa aponta que no sul do país, as taxas de internação por uso de drogas entre 2000 e 2012 mantiveram-se estáveis para os homens e aumentaram 3\% entre as mulheres ${ }^{11}$. Constatou-se ainda que, no período de 1999 a 2012, houve aumento relativo de $1.110 \%$ das internações psiquiátricas decorrentes do uso de múltiplas drogas, e o gasto médio por hospitalização passou de R\$ 451,23 em 1999 para R\$ 720,48 em 2012, com maiores gastos em internações por cocaína e crack ${ }^{12}$. Ressalta-se que os autores do estudo não consideraram o ajuste deflacionário dos gastos na moeda corrente brasileira nos anos analisados ${ }^{12}$.

Quanto aos resultados detectados no estado do Rio Grande do Sul, esses mostraram-se semelhantes aos de outro estudo conduzido na região, entre os anos de 2000 e 2011, no qual houve aumento de 125\% nas taxas de internações hospitalares devidas ao uso de álcool e outras drogas entre os homens e de $445 \%$ entre as mulheres ${ }^{24}$. Segundo os autores, o aumento expressivo das internações pode associar-se ao mascaramento da ocorrência de internações de longa permanência, por meio do fenômeno conhecido como porta giratória, no qual há o reingresso dos mesmos indivíduos em um curto espaço de tempo, e também ao comportamento contemporâneo do padrão de consumo de drogas e à inserção das mulheres em todos os setores da sociedade, que influenciaram a reformulação da saúde mental. Desse modo, apesar de haver oferta de serviços na comunidade, as hospitalizações em psiquiatria permanecem elevadas ${ }^{24}$.

Neste estudo, a região Norte diferiu-se do restante do país, demonstrando tendência crescente nas hospitalizações por uso de drogas em todo o período analisado. Trata-se de uma região propícia para o desenvolvimento de atividades ilícitas, especialmente para o tráfico de drogas, devido a sua posição geográfica estratégica para o escoamento de drogas produzidas pelos países vizinhos, como Colômbia, Peru e Bolívia, em virtude da vasta floresta tropical e dos grandes vazios demográficos ${ }^{25,26}$.

O Acre, estado da região Norte, consiste em um dos principais corredores para o tráfico de drogas no Brasil26. Ainda, entre 2005 e 2015, a população do estado cresceu 21\%, saltando de 661.427 para $803.513^{15}$. Esse evento demográfico, aliado aos problemas oriundos de uma região de fronteiras, bem como à inabilidade dos governos estaduais e federais para implementar políticas públicas sociais que atendam à demanda dos cidadãos, propiciou o desenvolvimento de "cracolândias". Isso gerou situações que atraem as pessoas para o tráfico, o consumo e o abuso de drogas, os quais podem suscitar mais hospitalizações ${ }^{25,26}$.

Ainda, aponta-se que na região Norte a cobertura dos CAPS é insuficiente, sugerindo dificuldades no manejo das crises sem o recurso hospitalar ${ }^{10}$. Nesse sentido, dados do Departamento de Informática do SUS (Datasus) demonstram que a região detém um dos menores quantitativos desse serviço no país, contando com apenas 187, sendo os estados do Acre e Amapá os menos favorecidos, com oito e sete estabelecimentos respectivamente ${ }^{15}$. A pequena disponibilidade de CAPS minimiza a efetividade da rede de atenção, com risco de retorno às práticas segregadoras. Municípios que possuem maior atuação dos serviços de base comunitária utilizam com menor frequência a hospitalização como recurso para o controle de agudizações dos transtornos mentais ${ }^{10}$.

Destaca-se que a atenção à saúde mental no Brasil enfrenta problemas associados à gestão dos serviços, às rein- 
ternações, à elevada demanda de usuários, à desarticulação da Rede de Atenção Psicossocial (RAPS) e à escassez de recursos humanos qualificados para atuar nesses serviços ${ }^{9}$. Não obstante, o poder público introduziu as comunidades terapêuticas na RAPS, promovendo o aumento do número de internações nesses dispositivos, também as de modalidade compulsória, cujas características são contrárias aos preceitos da Reforma Psiquiátrica, por violar os direitos humanos e promover o isolamento social, o estigma e hospitalizações longas (média de nove meses), com simultânea redução na expansão dos CAPS 6,27. Dessa forma, faz-se necessário que a RAPS seja reestruturada sob a perspectiva da Reforma Psiquiátrica, considerando a complexidade e o polimorfismo dos transtornos mentais e a trajetória de cada indivíduo, proporcionando, de fato, o atendimento integral e interdisciplinar e a reinserção social, não sucumbindo à visão biologista e hospitalocêntrica, a qual reduz o ser humano a doença e incapacidade ${ }^{8}$.

Quanto à diferença entre os sexos, há dados consistentes na literatura demonstrando que o consumo, o abuso e a dependência de drogas são significativamente mais altos entre os homens. Entretanto, nos últimos anos tal tendência tem se modificado, com acréscimo no envolvimento das mulheres com as drogas ${ }^{28,29}$. Um estudo desenvolvido na Espanha detectou que 58\% dos atendimentos em unidades de urgência e emergência hospitalares, relacionados ao uso de hipnóticos e sedativos, ocorreram entre o sexo feminino ${ }^{30}$.

As mulheres tendem a usar abusivamente os psicofármacos, estimulantes, sedativos e opiáceos ${ }^{3,28,29}$. No que se refere às drogas injetáveis, progridem mais rapidamente que os homens para a dependência e ainda as utilizam com maior frequência ${ }^{31}$. Entre os fatores de risco associados à dependência, os estudos salientam as questões genéticas, hormonais e, especialmente, ambientais, nas quais sofrem influência do companheiro, dos amigos e da família ${ }^{28,32}$. Mulheres usuárias têm maior probabilidade de desenvolver transtornos mentais, adquirir tuberculose e doenças sexualmente transmissíveis, sofrer violência física e sexual, e ter amenorreia, interrupção da gravidez, overdose e tentativas de suicídio ${ }^{32,33}$. Não obstante, são desassistidas em amplos aspectos, em consequência da condução falha das políticas públicas de saúde brasileiras, as quais reduzem os problemas femininos meramente aos cânceres de mama e colo do útero, e à fertilidade $^{22}$. Outro ponto que sustenta os achados desta pesquisa consiste no fato de que as mulheres procuram os serviços de saúde com maior frequência do que os homens, podendo-se detectar os sintomas de dependência logo no início e buscar a internação como alternativa para o seu tratamento ${ }^{28,33}$.

Resultado importante encontrado neste estudo foi o aumento das internações por drogas de abuso entre os idosos. Tal dado não se confirma na literatura internacional ${ }^{3,31}$, entretanto pode relacionar-se às características específicas da população brasileira. Corroborando esse achado, uma pesquisa conduzida com idosos diagnosticados com depressão e de- pendentes de drogas na região Sudeste do país identificou que 56\% possuíam entre 60 e 70 anos, as principais drogas de abuso foram o álcool (34\%) e o tabaco (26\%), seguido de sedativos e hipnóticos (10\%), e 48\% permaneceram entre 31 e 60 dias internados ${ }^{34}$

Vários elementos interagem e culminam no aumento das hospitalizações por uso de drogas em idosos, tais como a pobreza, os ambientes violentos, o contato íntimo com as drogas, a presença de depressão e/ou outros transtornos mentais, a solidão ou o abandono, distúrbios na autoimagem e a perda do papel social do idoso ${ }^{5}$. Destaca-se o fato de que, neste estudo, há elevada taxa de internações em pessoas de meia-idade (40 a 59 anos), levando a supor que, com o passar dos anos, mantenham o comportamento abusivo em relação às drogas, culminando, assim, no aumento de idosos dependentes de drogas.

Essa informação mostra-se inquietante, porquanto se associam os dispêndios conferidos pela droga às patologias de base frequentemente presentes entre os idosos, como diabetes mellitus, hipertensão arterial e depressão e a elevada incidência de câncer ${ }^{35}$. Assim, nesses indivíduos, os problemas advindos com o envelhecimento são agravados pela associação ao uso de drogas, levando ao maior número de hospitalizações e gastos com os serviços de saúde². Nessa perspectiva, faz-se necessário direcionar a assistência para essas pessoas, atentando para as suas especificidades, a fim de sensibilizá-las, tendo em vista os preceitos da redução de danos.

Nesse contexto, a dependência de drogas, essencialmente entre mulheres e idosos, possui alto impacto econômico e social, pois se agregam custos referentes à queda na produtividade, tratamento, reabilitação e redução dos danos, e até mesmo como combate à violência e ao tráfico ${ }^{1,5}$. Devido à magnitude desse fenômeno, as abordagens sobre o uso de drogas devem integrar amplos contextos, tais como o biopsicossocial, principalmente no tocante à estigmatização dos sujeitos, pois essa incide diretamente na assistência, desencorajando-os e afastando-os cada vez mais da sociedade e dos serviços de saúde 5 .

Destaca-se que, embora as pesquisas sobre o consumo de drogas entre as mulheres tenham se expandido no cenário internacional, elas ainda são incipientes no país. Os achados revelam-se alarmantes, tendo em vista a onerosa carga social e física conferida à mulher dependente de drogas. E ainda, quanto às internações entre os idosos, apesar de o Brasil seguir a tendência mundial quanto ao envelhecimento dos seus cidadãos, há poucos estudos que tratam do assunto, ressaltando a relevância da pesquisa. Nesse sentido, o estudo contribuiu para apontar o comportamento das internações e, assim, indicar os grupos com maior necessidade de intervenção.

Por fim, salienta-se que esta pesquisa possui limitações, por tratar-se de dados secundários oriundos do SIH/SUS, podendo haver erros de registro ou processamento, assim 
como não é possível identificar as reinternações. No entanto, o emprego de bases de dados secundárias como o SIH se faz imprescindível ao considerar as dimensões continentais do Brasil, além do fato de que esse sistema de informações vem sendo aperfeiçoado a cada ano, disponibilizando dados mais

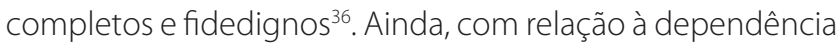
de drogas entre os idosos, não é possível afirmar que ela se iniciou na senilidade ou em outra faixa etária (e permaneceu ao longo dos anos), pois os dados apenas informam a idade em que ocorreu a hospitalização. Vale ressaltar outra limitação do estudo que é o uso de informações referentes apenas ao sistema público de saúde do país, pois no SIH não são registradas as internações ocorridas nas instituições privadas sem convênio com o SUS.

\section{CONCLUSÃO}

Os resultados demonstram tendência crescente das internações por uso de drogas até o ano de 2012, com posterior declínio para grande parte do país, com exceção da região Norte, que se manteve crescente. Detectou-se aumento das taxas de internações entre idosos e mulheres, com discreta diferença entre as regiões do país. Assim, o enfrentamento da dependência de drogas deve contemplar toda a população, atentando as especificidades de cada faixa etária e sexo, os quais podem envolver agravos de saúde que, ao não serem reconhecidos, comprometem a efetividade das ações, excluir grupos vulneráveis e não sensibilizar as pessoas. Ressalta-se a importância de estudos epidemiológicos a fim de identificar as características que incidem sobre a dependência de drogas. Dessa maneira, os profissionais, em conjunto com os gestores de saúde e as universidades, poderão traçar ações mais resolutivas, que visem ao enfrentamento da dependência de drogas sob a perspectiva da Reforma Psiquiátrica, oferecendo cuidado holístico e humanizado.

\section{CONTRIBUIÇÕES INDIVIDUAIS}

\section{Thamires Fernandes Cardoso da Silva Rodrigues e Ro-} sana Rosseto de Oliveira - Contribuíram com a concepção e delineamento do projeto, coleta, análise e interpretação dos dados, redação do artigo, revisão crítica e aprovação da versão final do artigo.

Maria das Neves Decesaro e Thais Aidar de Freitas Mathias - Contribuíram com a análise, interpretação dos dados, revisão crítica e aprovação da versão final do artigo.

\section{CONFLITOS DE INTERESSE}

Os autores declaram não haver conflitos de interesse de ordem pessoal, profissional, acadêmico, político ou financeiro.

\section{AGRADECIMENTOS}

O presente artigo foi realizado com o apoio da Coordenação de Aperfeiçoamento Pessoal de Nível Superior (Capes) - Código de Financiamento 001, por meio de bolsa de demanda social.

\section{REFERÊNCIAS}

1. United Nations Office on Drugs and Crime. World Drug Report 2017. Viena: New York. Disponível em: https://www.unodc.org/wdr2017/field/Booklet_1_EXSUM.pdf. Acesso em: 20 maio 2018.

2. Pavarin RM, Gambini D. Health status of users of the Bologna local health authority drug addiction treatment services: a study of hospital admissions in the period 2004-2013. Infez Med. 2015;23(1):5-11.

3. Lim D, Lee WK, Park H. Disability-Adjusted Life Years (DALYS) for Mental and Substance Use Disorders in the Korean Burden of Disease Study 2012. J Korean Med Sci. 2012;31(Suppl 2):191-9.

4. Chesney E, Goodwin GM, Fazel. Risks of all-cause and suicide mortality in mental disorders: a meta-review. World J Psychiatry. 2014;13(2):153-60.

5. Bokany V. Drogas no Brasil: entre a saúde e a justiça: proximidades e opiniões. São Paulo: Editora Fundação Perseu Abramo; 2015

6. Teixeira MB, Ramôa ML, Engstrom E, Ribeiro JM. Tensions between approach paradigms in public policies on drugs: an analysis of Brazilian legislation in 2000-2016. Ciênc Saúde Colet. 2017;22(5):1455-66.

7. Brasil. Ministério da Saúde. Lei no 10.216, de 6 de abril de 2001. Dispõe sobre a proteção e os direitos das pessoas portadoras de transtornos mentais e redireciona o modelo assistencial em saúde mental. Disponível em: http://hpm.org.br/wp-content/uploads/2014/09/ lei-no-10.216-de-6-de-abril-de-2001.pdf. Acesso em: 3 jun. 2018.

8. Coelho VAA, Volpe FM, Diniz SSL, Silva EM, Cunha CF. Alteration of profile of treatment of the public psychiatric hospitals of Belo Horizonte, Brazil, in the context of mental health care reform. Ciênc Saúde Coletiva. 2014;19(8):3605-16.

9. Júnior JMP, Santos RCA, Clementino FS, Oliveira KKD, Miranda FAN. Mental health policy in the context of psychiatric hospitals: Challenges and perspectives. Esc Anna Nery. 2016;20(1):83-9.

10. Onocko-Campos RT, Amaral CEM, Saraceno B, Oliveira BDC, Treichel CAS, Delgado PGG. Atuação dos Centros de Atenção Psicossocial em quatro centros urbanos no Brasil. Rev Panam Salud Publica. 2018;42:e113.

11. Balbinot AD, Horta RL, Costa JSD, Araújo RB, Poletto S, Teixeira MB. Hospitalization due to drug use did not change after a decade of the Psychiatric Reform. Rev Saúde Pública. 2016;50(26)

12. Zurita RCM, Melo EC, Oliveira RR, Latorre MRDO, Mathias TAF. Evolution of hospital spending with drug-related psychiatric hospital admissions. Rev Gaúcha Enferm. 2016;37(3):e53289.

13. Czyz EK, Berona J, King CA. Rehospitalization of Suicidal Adolescents in Relation to Course of Suicidal Ideation and Future Suicide Attempts. Psychiatr Serv. 2016;67(3):332-8.

14. Brasil. Ministério da Saúde. Relatório brasileiro sobre drogas. Secretaria Nacional de Políticas sobre Drogas (SENAD). Brasilia; 2009. Disponível em: http://www.escs.edu.br/arquivos/DrogasResumoExecutivo.pdf. Acesso em: 3 jun. 2018.

15. Brasil. Ministério da Saúde. Departamento de Informática do Sistema Único de Saúde (Datasus). Brasília; s.d. Disponível em: http://www2.datasus.gov.br/DATASUS/index. php?ared $=0203 \&$ id $=6926$. Acesso em: 3 jun. 2018.

16. Brasil. Ministério da Saúde. Resolução no 466, de 12 de dezembro de 2012. Brasília; 2012. Disponível em: http://conselho.saude.gov.br/resolucoes/2012/Res0466.pdf. Acesso em: 3 jun. 2018.

17. Toledo L, Góngora A, Bastos FIPM. On the sidelines of society: crack use, deviation, criminalization and social exclusion - a narrative review. Ciênc Saúde Coletiva. 2017;22(1):31-42.

18. Kendall CE, Boucher LM, Mark AE, Martin A, Marshall Z, Boyd R, et al. A cohort study examining emergency department visits and hospital admissions among people who use drugs in Ottawa, Canada. Harm Reduct J. 2017;14(16). 
19. Nguyen TL, Boudemaghe T, Leguelinel-Blache G, Eiden C, Kinowski JM, Mach YL, et al. Identifying Life-Threatening Admissions for Drug Dependence or Abuse (ILIADDA): Derivation and Validation of a Model. Sci Rep. 2017;7:44428.

20. Duarte SL, Garcia MLT. Psychiatric Reform: the path of psychiatric beds reduction in Brazil. Emancipação. 2013;13(1):39-54.

21. Brasil. Ministério da Saúde. Políticas de saúde mental: baseado no curso políticas públicas de saúde mental, do CAPS. São Paulo; 2013. Disponível em: http://www.saude.sp.gov. br/resources/instituto-de-saude/homepage/outras-publicacoes/politicas_de_saude_ mental_capa_e_miolo_site.pdf. Acesso em: 4 jun. 2018.

22. França $A M B$, Silva JM0. Women in vulnerable situation: a view from perspective of social phenomenology. Rev Enferm UFPE. 2016;10(Suppl.2):875-9.

23. Monteriro Neto A. Desigualdades regionais no Brasil: características e tendências recentes. BRU [Internet]. 2014 [citado em 22 de nov de 2018];09:67-81. Disponível em: http://ipea. gov.br/agencia/images/stories/PDFs/boletim_regional/141211_bru_9_web_cap8.pdf. Acesso em: 4 jun. 2019.

24. Horta RL, Costa JSD, Balbinot AD, Watte G, Teixeira VA, Poletto S. Psychiatric hospitalizations in the Rio Grande do Sul State (Brazil) from 2000 to 2011. Rev Bras Epidemiol. 2015;18(4):918-29.

25. Aguilara MAB, Gonçalves JP.Drug use in Adolescent in Border Region: Interface with Human Rights. Rev Adol Confl. 2017;(16):26-32.

26. Souza AJS. Drug trafficking: coping and public policy - an overview Rondônia. Cadernos de Dereito Actual. 2015;(3):277-93.

27. Pereira MO, Oliveira MAF, Pinho PH, Claro HG, Gonçalves AM, Reinaldo MAS. What is the emphasis of Brazilian drug policy: resocialization or internment? Esc Anna Nery. 2017;21(3):e20170044.
28. Lal R, Deb KS, Kedia S. Substance use in women: Current status and future directions. Indian J Psychiatry. 2015;57(2):275-85.

29. Minutillo A, Pacifici R, Scaravelli G, Luca R, Palmi I, Mortali C, et al. Gender disparity in addiction: an Italian epidemiological sketch. Ann Ist Super Sanità. 2016;52(2):176-83.

30. González VR, Martín SR, Prado GRL, Moreno MFM, Miranda AV. Urgencias hospitalarias asociadas al consumo de hipnóticos y sedantes, Castilla y León, 2009-2013. Rev Espan Salud Pública. 2013;90(25).

31. Khuart OTH, Marrow M, Nguyen TNN, Armstrong G. Social context, diversity and risk among women who inject drugs in Vietnam: descriptive findings from a cross-sectional survey. Harm Reduction J. 2015;12(35):1-8.

32. Brasil. Ministério da Justiça. 0 uso de substâncias psicoativas no Brasil: módulo 1. Secretaria Nacional de Políticas sobre Drogas. Brasília; 2014. Disponível em: http://www. supera.senad.gov.br/wp-content/uploads/2016/03/SUP7_Mod1.pdf. Acesso em: 5 jun. 2018.

33. Wardle MC, Marcus BA, Wit H. A Preliminary Investigation of Individual Differences in Subjective Responses to D-Amphetamine, Alcohol, and Delta-Tetrahydrocannabinol Using a Within-Subjects Randomized Trial. Plos One. 2015;10(10):e0140501.

34. Cantão L, Fonseca LLK, Silva TIM, Oliveira M, Oliveira VC, Machado RM. Sociodemographic and clinical profile of elderly people with depression and the use of psychoactive drugs. Rev Rene. 2015;16(3):355-62.

35. Brasil. Ministério da Saúde. Estatuto do Idoso. Brasília; 2013. Disponível em: http://bvsms. saude.gov.br/bvs/publicacoes/estatuto_idoso_3edicao.pdf. Acesso em: 5 jun. 2018.

36. Hatisuka MFB, Arruda GO, Fernandes CAM, Marcon SS. Tendency analysis of admission rates for bacterial pneumonia in children and adolescents. Acta Paul Enferm. 2015;28(4):294-300. 